\title{
ELECTROMAGNETIC WAVE SCATTERING AND CONVERSION BY LOW-FREQUENCY COLLECTIVE FLUCTUATIONS IN MAGNETIZED PLASMAS
}

\author{
A. G. Sitenko \\ Bogolyubov Institute for Theoretical Physics \\ 14-b Metrolohichna Str., UA-03143, Kyiv, Ukraine
}

(Received 10 September 1999)

\begin{abstract}
The problem of electromagnetic wave scattering in a plasma placed in a strong external magnetic field and its possible applications for plasma diagnostics is discussed. The scattering is associated both with longitudinal field fluctuations (charge density fluctuations) and with transverse electromagnetic fluctuations. Electromagnetic wave scattering by magnetic fluctuations is accompanied by wave conversion. Low-frequency fluctuation spectra of electric and magnetic fields are calculated for a plasma with a strong external magnetic field. Incoherent fluctuations and fluctuations associated with the excitation of collective modes (Alfven and magnetosonic waves) are singled out. The spectral distributions of the electric and magnetic field fluctuations in nonequilibrium plasmas are considered. Fluctuations are also considered in a plasma with large-scale turbulent pulsations. The wave scattering cross-section is calculated and the conditions are revealed, under which conversion is the dominant process. Peculiar features of scattered waves spectral distribution depending on the parameters that characterize plasma state are treated.
\end{abstract}

Key words: magnetized plasma, Alfven and magnetosonic fluctuations, scattering, conversion, electromagnetic wave.

PACS numbers: 52.25. Gj

\section{INTRODUCTION}

The study of electromagnetic wave scattering spectra (both in laser and centimeter wave ranges) is an efficient method of plasma diagnostics in laboratory fusion research devices as well as in the near and outer space. Electromagnetic wave scattering is caused by fluctuations of charged particle density and other plasma parameters: current density, electric and magnetic fields. Spectra of scattered waves provide information on the density and temperature distributions in the plasma. A peculiarity of electromagnetic wave scattering in plasmas is coherent scattering by collective plasma excitations combination scattering, that occurs along with Thompson incoherent scattering by individual plasma particles. Wave scattering by collective plasma fluctuations, in particular, makes it possible to find relative concentrations of charged particles and temperatures of individual plasma components. The phenomenon of electromagnetic wave combination scattering by collective plasma excitations was considered for the first time in [1]. As distinct from the wave scattering by fluctuations of charged particle density, interaction of the incident wave and the magnetic field fluctuations may be accompanied by the electromagnetic wave conversion, i.e., transformation of an ordinary electromagnetic wave into an extraordinary one or vice versa. Though the scattering cross-section is much greater than the conversion cross-section, under certain condition one can distinguish conversion from scattering and find the spectral distribution of converted waves. Studies of converted wave spectral distributions provide information on plasma parameters other than those associated with wave scattering, in particular on the magnetic field fluctuation intensity distribution. Increased activities in the controlled fusion research and employment of magnetic plasma confinement systems require improved methods of plasma state analysis. It is obvious that information on the plasma state can be drawn from experimental data only provided the theoretical spectra of magnetic and electric field fluctuations in the plasma are available.

\section{PLASMA WITH STRONG MAGNETIC FIELD}

Electromagnetic plasma properties are completely described in terms of the plasma dielectric permittivity tensor $\varepsilon_{i j}(\omega, \mathbf{k})$ which depends on the frequency $\omega$ and the wave vector $\mathbf{k}$. The dielectric permittivity tensor of a plasma with external magnetic field was calculated in the kinetic approximation in $[2,3]$.

In order to consider a plasma with strong external magnetic field $\mathbf{B}_{0}$ we introduce a dimensionless parameter,

$$
q^{2}=\frac{k^{2} s^{2}}{\omega_{B_{i}}^{2}} \ll 1,
$$

where $s=\sqrt{\frac{3 T}{m}}$ is the electron thermal velocity ( $T$ is the plasma temperature, $m$ is the electron mass) and $\omega_{B_{i}}=\frac{e B_{0}}{M c}$ is the ion cyclotron frequency ( $e$ and $M$ are the ion charge and mass, respectively). We restrict the consideration to the spectral range of frequencies $\omega$ lower 
than the electron cyclotron frequency $\omega_{B_{e}}=\frac{e B_{0}}{m c}$, i.e., $\omega \ll \omega_{B_{e}}$, and employ approximate expressions for the plasma dielectric permittivity components, obtained under the assumption that $\frac{m}{M} \ll 1$ and expanded in power series of the small parameter $q^{2}$. In the coordinate system with the $z$-axis along the vector $\mathbf{B}_{0}$ and the $x$-axis in the plane of vectors $\mathbf{k}$ and $\mathbf{B}_{0}$, the components of the dielectric permittivity tensor $\varepsilon_{i j}(\omega, \mathbf{k})$ take the form

$$
\begin{aligned}
& \varepsilon_{11}=\frac{1}{2} \frac{\omega_{p_{i}}^{2}}{\omega^{2}}\left\{\omega\left(\frac{1}{\omega_{B_{i}}-\omega} \varphi\left(z_{1}\right)-\frac{1}{\omega_{B_{i}}-\omega}\right)+i \sqrt{\pi} \mu z e^{-z_{1}^{2}}\right\}, \\
& \varepsilon_{22}=\varepsilon_{11}-\frac{m}{M} \varepsilon_{0}\left\{1+\varphi(z)-i \sqrt{\pi} z e^{-z^{2}}\right\} \frac{\operatorname{tg}^{2} \vartheta}{z^{2}}, \\
& \varepsilon_{33}=3 \frac{M}{m} \varepsilon_{0}\left\{1-\varphi(z)-\varphi(\mu z)+i \sqrt{\pi} z\left(e^{-z^{2}}+\mu e^{-\mu^{2} z^{2}}\right)\right\} \frac{1}{q^{2} \cos ^{2} \vartheta}, \\
& \varepsilon_{12}=i\left\{\varepsilon_{11}-\frac{\omega_{p_{i}}^{2}}{\omega_{B_{i}}\left(\omega_{B_{i}}+\omega\right)}\right\}, \\
& \varepsilon_{23}=-i \varepsilon \frac{\omega_{B_{i}}}{\omega}\left\{1-\varphi(z)+i \sqrt{\pi} z e^{-z^{2}}+\frac{1}{2}\left(1-\varphi\left(z_{1}\right)+i \sqrt{\pi} z_{1} e^{-z_{1}^{2}}\right)\right\} \operatorname{tg} \vartheta \\
& \varepsilon_{13}=\frac{1}{2} \varepsilon_{0} \frac{\omega_{B_{i}}}{\omega}\left\{1-\varphi\left(z_{1}\right)+i \sqrt{\pi} z_{1} e^{-z_{1}^{2}}\right\} \operatorname{tg} \vartheta .
\end{aligned}
$$

Here $\vartheta$ is the angle formed by the vectors $\mathbf{k}$ and $\mathbf{B}_{0} ; \varepsilon_{0}=\frac{\omega_{p_{i}}^{2}}{\omega_{B_{i}}^{2}}$, where $\omega_{p_{i}}^{2}=\frac{4 \pi n_{n} e^{2}}{M}$ is the square of the ion plasma frequency (we assume that $\varepsilon_{0} \gg 1$ ); $\mu^{2}=\frac{M}{m} ; z=\sqrt{\frac{3}{2}} \frac{\omega}{k s \cos \vartheta}$ is the dimensionless frequency and $z_{1}=\sqrt{\frac{3}{2} \frac{M}{m}} \frac{\left|\omega_{B_{i}}-\omega\right|}{k s \cos \vartheta}$.

In (1), only the first terms of the $q^{2}$-expansions of general expressions for the permittivity tensor of a plasma with external magnetic field are retained. We note that individual components of the permittivity tensor (1) differ in the order of magnitude with respect to the parameter $q^{2}$. The leading term of the expansion of the component $\varepsilon_{33}$ is proportional to $q^{-2}$ (for very low frequencies, one has to take into account the ion motion in $\left.\varepsilon_{33}\right)$, and those of the components $\varepsilon_{11}$ and $\varepsilon_{22}$ are of the order of one.

\section{EIGENWAVES OF A PLASMA WITH STRONG EXTERNAL MAGNETIC FIELD}

We introduce the dispersion tensor $\Lambda_{i j}(\omega, \mathbf{k})$ :

$$
\Lambda_{i j}(\omega, \mathbf{k})=\varepsilon_{i j}(\omega, \mathbf{k})-\left(\delta_{i j}-\frac{k_{i} k_{j}}{k^{2}}\right) \eta^{2}
$$

where $\eta^{2}=\frac{k^{2} c^{2}}{\omega^{2}}$ is the square of the wave refraction index and write its determinant in the form

$$
\begin{aligned}
\Lambda(\omega, \mathbf{k}) & =\varepsilon_{33}\left\{\left(\eta^{2} \cos ^{2} \vartheta-\varepsilon_{11}\right)\left(\eta^{2}-\varepsilon_{22}-\frac{\varepsilon_{23}^{2}}{\varepsilon_{33}}\right)\right. \\
& +\varepsilon_{12}^{2}+\frac{\eta^{2}}{\varepsilon_{33}}\left[\left(\eta^{2}-\varepsilon_{22}\right) \varepsilon_{11} \sin ^{2} \vartheta\right. \\
& \left.\left.+2 \varepsilon_{12} \varepsilon_{23} \sin \vartheta \cos \vartheta-\varepsilon_{12}^{2} \sin ^{2} \vartheta\right]\right\} .
\end{aligned}
$$

The large quantity $\varepsilon_{33}=0\left(q^{-2}\right)$ is put before the curly brackets for convenience. The two first addends between the curly brackets are of the order of one, all the other terms are of the order of $q^{2}$.

The dispersion equation that determines the frequencies and damping rates of plasma eigenwaves reduces to the condition

$$
\Lambda(\omega, \mathbf{k})=0 .
$$

The eigenfrequencies are determined by the real part of the dispersion equation in which thermal corrections contained in the expressions for the dielectric permittivity components may be neglected, while the wave damping rate is determined by the imaginary part of the dispersion determinant (3) that is given rise to by the thermal effects.

In the long-wave length limit, for small values of the parameter $\xi^{2}=\frac{k^{2} v_{A}^{2}}{\omega_{B_{i}}^{2}}\left(v_{A}=\frac{B_{0}}{\sqrt{4 \pi n_{0} M}}\right.$ is the Alfven speed $)$, 


\section{A. G. SITENKO}

the dispersion equation (4) has two different solutions in the low-frequency range which correspond to the Alfven wave (A) and the fast magnetosonic wave (S) [4].

The frequency and the damping rate of the Alfven wave are defined by

$$
\omega_{A}=k v_{A} \cos \vartheta, \quad \gamma_{A}=\frac{1}{2} \omega_{A} P^{\prime \prime}\left(\omega_{A}\right)
$$

where the notation is introduced:

$$
\begin{aligned}
P^{\prime \prime}(\omega) & =\frac{\sqrt{\pi}}{3} \frac{m}{M} q^{2}\left\{\frac{\omega^{4}}{\left(k^{2} v_{A}^{2}-\omega^{2}\right)^{2}}\right. \\
& \left.+\frac{k^{2} v_{A}^{2}}{\omega^{2}} \frac{\cos ^{2} \vartheta}{[1-\varphi(z)]^{2}+\pi z^{2} e^{-2 z^{2}}}\right\} z e^{-z^{2}} .
\end{aligned}
$$

The electric field vector of the Alfven wave is perpendicular to $\mathbf{B}_{0}$ and lies in the plane of vectors $\mathbf{k}$ and $\mathbf{B}_{0}$, hence for the polarization vector we have $\mathbf{e}^{(A)}=(1,0,0)$.

The frequency and the damping rate of the fast magnetosonic wave are given by

$$
\omega_{S}=k v_{A}, \quad \gamma_{S}=\frac{1}{2} \omega_{S} \Psi^{\prime \prime}\left(\omega_{S}\right)
$$

where

$$
\Psi^{\prime \prime}(\omega)=\frac{\sqrt{\pi}}{2} \frac{m}{M} \operatorname{tg}^{2} \vartheta \frac{e^{-z^{2}}}{z}, \quad z^{2}=\frac{3}{2} \frac{\omega^{2}}{k^{2} s^{2} \cos ^{2} \vartheta} .
$$

The electric field vector of the fast magnetosonic wave is perpendicular to the plane of vectors $\mathbf{k}$ and $\mathbf{B}_{0}$, hence $\mathbf{e}^{(S)}=(0,1,0)$.

As the eigenwave frequency approaches the ioncyclotron frequency $\omega_{B_{i}}$, the refraction index of the Alfven wave tends to infinity, whereas the refraction index of the magnetosonic wave remains finite. For $\omega \rightarrow$ $\omega_{B_{i}}$, the cyclotron damping of the Alfven wave becomes so strong that propagation of this wave becomes impossible.

\section{FLUCTUATIONS IN EQUILIBRIUM PLASMAS}

According to the fluctuation-dissipation theorem, the spectral distribution of electric field fluctuations is given by

$$
\left\langle E_{i} E_{j}\right\rangle_{\mathbf{k} \omega}=4 \pi i \frac{T}{\omega}\left\{\Lambda_{j i}^{-1}-\left(\Lambda_{i j}^{-1}\right)^{*}\right\}
$$

where $T$ is the plasma temperature and $\Lambda_{i j}^{-1}$ is the inverse of the dispersion tensor (2). We introduce the algebraic complement $\lambda_{i j}$ of the tensor (2),

$$
\lambda_{i j} \Lambda_{j k}=\Lambda \delta_{j k}, \quad \Lambda \equiv\left\|\Lambda_{i j}\right\|,
$$

then the inverse tensor may be represented in the form

$$
\Lambda_{i j}^{-1}=\frac{\lambda_{i j}}{\Lambda}
$$

The spectral distribution of magnetic field fluctuations is related to the electric field fluctuation distribution as given by

$$
\left\langle B_{i} B_{j}\right\rangle_{\mathbf{k} \omega}=\eta^{2} \epsilon_{i k m} \epsilon_{j l n} \frac{k_{k} k_{l}}{k^{2}}\left\langle E_{m} E_{n}\right\rangle_{\mathbf{k} \omega}
$$

where $\epsilon_{i k m}$ is a completely antisymmetric third-rank tensor.

\section{SPECTRAL DISTRIBUTIONS OF ELECTRIC FIELD FLUCTUATIONS}

Making use of expression (1) for components of the dielectric permittivity tensor in the case $q^{2} \ll 1$ we give the algebraic complement tensor (2) in the form

$$
\lambda_{i j}=\lambda_{i j}^{\text {long }}-\varepsilon_{33}\left(\begin{array}{lll}
\eta^{2}-\varepsilon_{22}-\frac{\varepsilon_{23}^{2}}{\varepsilon_{33}} & 0 & 0 \\
0 & \eta^{2} \cos ^{2} \vartheta-\varepsilon_{11} & 0 \\
0 & 0 & 0
\end{array}\right),
$$

where $\lambda_{i j}^{\text {long }}=\eta^{4} \frac{k_{i} k_{i}}{k^{2}}$ is longitudinal part of tensor $\lambda_{i j}$ which we can disregard in the range of frequencies $\omega>\sqrt{\frac{m}{3 M}} q k v_{A} \cos \vartheta$. Thus, only the components $\lambda_{11}$ and $\lambda_{22}$ are of the same order of magnitude in the limiting case $q^{2} \rightarrow 0$ (all the other components $\lambda_{33}, \lambda_{12}, \lambda_{13}$ and $\lambda_{23}$ and are of higher orders of smallness). Therefore, the spectral distribution of electric field fluctuations in a plasma with strong external magnetic field in the range of frequencies much lower than the ion cyclotron frequency $\left(\omega \ll \omega_{B_{i}}\right)$ is determined by the expression:

$$
\left\langle E_{i} E_{j}\right\rangle_{\mathbf{k} \omega}=8 \pi T \frac{v_{A}^{2}}{c^{2}} \omega\left\{e_{i}^{(A)} e_{j}^{(A)} \frac{\omega^{2} P^{\prime \prime}}{\left(\omega^{2}-k^{2} v_{A}^{2} \cos ^{2} \vartheta\right)^{2}+\left(\omega^{2} P^{\prime \prime}\right)^{2}}+e_{i}^{(S)} e_{j}^{(S)} \frac{\psi^{2} \Psi^{\prime \prime}}{\left(\omega^{2}-k^{2} v_{A}^{2}\right)^{2}+\left(\omega^{2} \Psi^{\prime \prime}\right)^{2}}\right\}
$$


We see that the spectral distribution of fluctuations has two maxima associated with Alfven and magnetosonic fluctuation oscillations. These maxima are manifested in different components of the fluctuation spectral distribution tensor because of differing polarizations of the fluctuation electric field vectors. The maximum associated with the Alfven fluctuation oscillations occurs in the spectral distribution of the component $\left\langle E_{1}^{2}\right\rangle_{\mathbf{k} \omega}$ whereas the one corresponding to the magnetosonic fluctuation oscillations is manifested, respectively, in the spectral distribution of the component $\left\langle E_{2}^{2}\right\rangle_{\mathbf{k} \omega}$. Numerical calculations of the spectral distributions $\left\langle E_{1}^{2}\right\rangle_{\mathbf{k} \omega}$ and $\left\langle E_{2}^{2}\right\rangle_{\mathbf{k} \omega}$ were performed in [5] for the values of the parameters $\Theta^{2} \equiv \frac{3}{2} \frac{v_{A}^{2}}{s^{2}}$ and $q^{2}$ which correspond to the actual conditions of real plasma with the concentration $n_{0}=1.2 \cdot 10^{14} \mathrm{~cm}^{-3}$, temperature $T=10^{4} \mathrm{eV}$, and external magnetic field $B_{0}=3.4 \cdot 10^{4} \mathrm{Gs}$.

The magnetosonic perturbation is transverse (the polarization vector of the fast magnetosonic wave is perpendicular to the wave vector, $\mathrm{e}^{(S)} \mathbf{k}=0$ ); the electric field of Alfven perturbations has a longitudinal component as well. To find the spectral distribution of the longitudinal electric field, we multiply the total distribution (11) by $\frac{k_{i} k_{j}}{k^{2}}$ and thus separate out the longitudinal component

$$
\left\langle E_{l}^{2}\right\rangle_{\mathbf{k} \omega}^{(A)}=8 \pi T \frac{v_{A}^{2}}{c^{2}} \omega \sin ^{2} \vartheta \frac{\omega^{2} P^{\prime \prime}}{\left(\omega-k^{2} v_{A}^{2} \cos ^{2} \vartheta\right)^{2}+\left(\omega^{2} P^{\prime \prime}\right)^{2}} .
$$

The spectral distribution of charge density fluctuations associated with Alfven perturbations in the plasma is determined by the relation

$$
\left\langle\rho^{2}\right\rangle_{\mathbf{k} \omega}^{(A)}=\frac{k^{2}}{16 \pi^{2}}\left\langle E_{l}^{2}\right\rangle_{\mathbf{k} \omega}^{(A)}
$$

The spectral distribution of the charge density fluctuations (13) has a maximum for frequencies corresponding to Alfven fluctuation waves and a broad maximum in the range of low frequencies associated with incoherent plasma fluctuations.

In the low-frequency range $z^{2} \ll \frac{m}{M}$, the spectral distribution (13) takes the form

$$
\begin{aligned}
& \left\langle\rho^{2}\right\rangle_{\mathbf{k} \omega}^{(A)}=\frac{1}{6} \sqrt{\frac{\pi}{2}} \frac{e^{2} n_{0}}{\omega_{P_{i}}} \frac{\omega^{2}}{\omega_{P_{\epsilon}^{2}}} \frac{s^{2}}{v_{A}^{2}} k a \sin \vartheta t g^{3} \vartheta e^{-\frac{M}{m} z^{2}} \\
& a^{2}=\frac{T}{4 \pi n_{0} e^{2}}
\end{aligned}
$$

We note that intensities of both charge density and longitudinal electric field fluctuations $\left\langle\rho^{2}\right\rangle_{\mathbf{k} \omega}^{(A)}$ and $\left\langle E_{l}^{2}\right\rangle_{\mathbf{k} \omega}^{(A)}$ tend to zero in the low-frequency range.

In the low-frequency domain dominant contribution to the incoherent charge density fluctuations is associated with the longitudinal electric field. The spectral distribution of such incoherent fluctuations is described by

$$
\left\langle\rho^{2}\right\rangle_{\mathbf{k} \omega}=\frac{1}{2} \sqrt{\frac{\pi}{2}} \frac{e^{2} n_{0}}{\omega_{P_{i}}} k^{3} a^{3} \frac{e^{-\frac{M}{m} z^{2}}}{\cos \vartheta}, \quad z^{2} \ll \frac{m}{M}
$$

The spectrum of electron density incoherent fluctuations in the low-frequency domain is as follows

$$
\left\langle\delta n_{e}^{2}\right\rangle_{\mathbf{k} \omega}=\frac{1}{2} \sqrt{\frac{3 \pi}{2}} \sqrt{\frac{M}{m}} \frac{n_{0}}{k s \cos \vartheta} e^{-\frac{M}{m} z^{2}}, \quad z^{2} \ll \frac{m}{M}
$$

As the wave number increases, the maxima of electric field fluctuation spectra are shifted to the ion cyclotron frequency range and separation of Alfven and magnetosonic fluctuation excitations disappears in individual components of the spectral distribution. The components $\left\langle E_{1}^{2}\right\rangle_{\mathbf{k} \omega}$ and $\left\langle E_{2}^{2}\right\rangle_{\mathbf{k} \omega}$ of the spectral distribution of electric field fluctuations in frequency range $\omega \sim \omega_{B_{i}}$ were calculated in [5] as functions of the dimensionless frequency $\widetilde{\omega} \equiv \frac{\omega}{\omega_{B_{i}}}$ for different values of parameter $\xi$. Each spectral distribution $\left\langle E_{1}^{2}\right\rangle_{\mathbf{k} \omega}$ and $\left\langle E_{2}^{2}\right\rangle_{\mathbf{k} \omega}$ has four maxima. Two maxima in the low-frequency range $\widetilde{\omega}<0.01$ and in the range of ion cyclotron frequency $\widetilde{\omega} \approx 1\left(\omega \approx \omega_{B_{i}}\right)$ are associated with incoherent fluctuations. Two narrow maxima correspond to the Alfven (at the frequency $\omega_{A}$ ) and magnetosonic (at the frequency $\omega_{S}$ ) collective fluctuation oscillations $\left(\omega_{A}<\omega_{S}\right)$. These maxima are by several orders of magnitude higher than the maxima associated with incoherent fluctuations. Position and magnitude of the maxima which correspond to the Alfven and magnetosonic fluctuation oscillations depend on parameter $\xi$. When $\xi$ increases the $\omega_{A}$ and $\omega_{S}$ are also increases (to narrow maxima are shifted to the range of the higher frequency), however $\omega_{A}<\omega_{B_{i}}\left(\widetilde{\omega}_{A} \equiv \frac{\omega_{A}}{\omega_{B_{i}}}<1\right)$. When $\xi=0.7516$ the maximum associated with magnetosonic collective fluctuations is located at the frequency $\widetilde{\omega}_{S}=1$ (incoherent maximum is also situated at this frequency). When $\xi=1$ the maximum associated with magnetosonic collective fluctuations lies outside the frequency range $\omega \approx \omega_{B_{i}}$. For the values of parameter $\xi=0.2 ; 0.4$ the maximum associated with magnetosonic fluctuation oscillations is by several orders of magnitude higher than maximum associated with Alfven fluctuation oscillations. The magnitude of the maximum in the range of ion cyclotron frequency $\widetilde{\omega} \approx 1\left(\omega \approx \omega_{B_{i}}\right)$ which associated with incoherent fluctuations is increasing when parameter $\xi$ is increasing.

In the ion cyclotron resonance range, $\left(\frac{\omega_{B_{i}}-\omega}{\omega_{B_{i}}}\right)^{2} \ll$ $\frac{2}{3} \frac{m}{M} q^{2} \cos ^{2} \vartheta$, both electric field fluctuation spectra $\left\langle E_{1}^{2}\right\rangle_{\mathbf{k} \omega}$ and $\left\langle E_{2}^{2}\right\rangle_{\mathbf{k} \omega}$ have a maximum associated with incoherent cyclotron fluctuations. It is given by

$$
\begin{aligned}
\left\langle E_{1}^{2}\right\rangle_{\mathbf{k} \omega} & =2 \pi \sqrt{6 \pi \frac{M}{m}} \frac{T}{\omega_{P_{i}}} \frac{\omega_{B_{i}}}{\omega_{P_{i}}} \frac{c}{s} \psi(\xi) e^{-z_{1}^{2}} \\
\left\langle E_{2}^{2}\right\rangle_{\mathbf{k} \omega} & =\cos ^{2} \vartheta\left\langle E_{1}^{2}\right\rangle_{\mathbf{k} \omega},
\end{aligned}
$$




\section{A. G. SITENKO}

where

$$
\begin{gathered}
z_{1}^{2}=\frac{3}{2} \frac{M}{m} \frac{\left(\omega_{B_{i}}-\omega\right)^{2}}{k^{2} s^{2} \cos ^{2} \vartheta} \quad \text { and } \quad \psi(\xi)=\frac{1}{\xi^{5} \cos ^{5} \vartheta} \\
\text { for } \quad \xi^{2}>1 .
\end{gathered}
$$

\section{SPECTRAL DISTRIBUTION OF MAGNETIC FIELD FLUCTUATIONS}

Making use of relation (9) between the distributions of electric and magnetic field fluctuations and the spectral distribution of electric field fluctuations (11), we find the spectral distribution of long-wavelength fluctuations $\left(k v_{A} \ll\right.$ $\omega_{B_{i}}$ ) of magnetic field in a plasma with strong external magnetic field to be given by

$$
\begin{aligned}
\left\langle B_{i} B_{j}\right\rangle_{\mathbf{k} \omega} & =8 \pi \frac{T}{\omega} k^{2} v_{A}^{2}\left\{b_{i}^{(A)} b_{j}^{(A)} \frac{\omega^{2} P^{\prime \prime} \cos ^{2} \vartheta}{\left(\omega^{2}-k^{2} v_{A}^{2} \cos ^{2} \vartheta\right)^{2}+\left(\omega^{2} P^{\prime \prime}\right)^{2}}+b_{i}^{(S)} b_{j}^{(S)} \frac{\omega^{2} \Psi^{\prime \prime}}{\left(\omega^{2}-k^{2} v_{A}^{2}\right)^{2}+\left(\omega^{2} \Psi^{\prime \prime}\right)^{2}}\right\}, \\
\omega & \ll \omega_{B_{i}},
\end{aligned}
$$

where we introduced the magnetic field polarization vectors for the Alfven and magnetosonic waves; $\mathbf{b}^{(A)}=(0,1,0)$, $\mathbf{b}^{(S)}=(-\cos \vartheta, 0, \sin \vartheta)$.

Similarly to the spectral distribution of electric field fluctuations, the spectral distribution of magnetic field fluctuations (18) has two maxima given rise to by Alfven and magnetosonic fluctuation oscillations and a low-frequency maximum associated with incoherent fluctuations. As in the case of electric field fluctuations, differing polarizations of Alfven and magnetosonic waves are responsible for the fact that relevant magnetic field fluctuation maxima are manifested in different components of the spectral distribution tensor. The maximum associated with the Alfven excitations appears in the spectral distribution of the component $\left\langle B_{2}^{2}\right\rangle_{\mathbf{k} \omega}$ whereas the maximum corresponding to the magnetosonic fluctuations is manifested in the spectral distributions of $\left\langle B_{1}^{2}\right\rangle_{\mathbf{k} \omega},\left\langle B_{3}^{2}\right\rangle_{\mathbf{k} \omega},\left\langle B_{1} B_{3}\right\rangle_{\mathbf{k} \omega}$ and $\left\langle B_{3} B_{1}\right\rangle_{\mathbf{k} \omega}$.

As distinct from the case of electric field fluctuations, the maximum of incoherent fluctuations of the magnetic field corresponds to the zero frequency. Bearing in mind that ion motion should be allowed for in the case of very low frequencies $\left(z^{2} \ll \frac{m}{M}\right)$, we obtain the expressions for the spectral distributions of incoherent Alfven and magnetosonic fluctuations, i.e.,

$$
\begin{gathered}
\left\langle B_{2}^{2}\right\rangle_{\mathbf{k} \omega}^{(A)}=2 \pi \sqrt{\frac{\pi}{6} \frac{m}{M}} \frac{T}{k s} q^{2} \sin \vartheta \operatorname{tg} \vartheta e^{-\frac{M M}{m} z^{2}}, \quad \omega \ll k v_{a} \cos \vartheta \\
\left\langle B_{i} B_{j}\right\rangle_{\mathbf{k} \omega}^{(S)}=b_{i}^{(S)} b_{j}^{(S)} 8 \pi \sqrt{\frac{2 \pi}{3} \frac{m}{M}} \frac{T}{k v_{A}} \sin \vartheta \operatorname{tg} \vartheta e^{-\frac{M}{m} z^{2}}, \quad \omega \ll k v_{a} .
\end{gathered}
$$

The widths of the distributions are determined by the ion thermal velocity.

\section{FLUCTUATIONS IN NONEQUILIBRIUM PLASMAS}

Electromagnetic fluctuations in a nonequilibrium plasma are determined by the spectral distribution of the Langevin current along with the dielectric permittivity tensor.

The spectral distribution of electric field fluctuations in a nonequilibrium plasma is determined by the expression

$$
\left\langle E_{i} E_{j}\right\rangle_{\mathbf{k} \omega}=\frac{16 \pi}{\omega^{2}} \frac{1}{|\Lambda|^{2}} \lambda_{i k}^{*} \lambda_{j l}\left\langle j_{k} j_{l}\right\rangle_{\mathbf{k} \omega}^{0}
$$

and the spectral distribution of magnetic field fluctuations in a nonequilibrium plasma is given by 


$$
\left\langle B_{i} B_{j}\right\rangle_{\mathbf{k} \omega}=\frac{16 \pi^{2}}{\omega^{2}} \frac{\eta^{2}}{|\Lambda|^{2}} \epsilon_{i k m} \epsilon_{j l n} \frac{k_{k} k_{l}}{k^{2}} \lambda_{m r}^{*} \lambda_{n s}\left\langle j_{r} j_{s}\right\rangle_{\mathbf{k} \omega}^{0}
$$

The spectral distribution of current density fluctuations in the plasma disregarding the Coulomb interaction of charged particles is usually taken for the spectral distribution of the Langevin current:

$$
\left\langle j_{i} j_{j}\right\rangle_{\mathbf{k} \omega}^{0}=e^{2} \int_{-\infty}^{\infty} d t e^{i \omega t} \int d \mathbf{v} \int d \mathbf{v}^{\prime} v_{i} v_{j} W_{\mathbf{k}}\left(\mathbf{v}, \mathbf{v}^{\prime} ; t\right) f_{0}(\mathbf{v})
$$

where $W_{\mathbf{k}}\left(\mathbf{v}, \mathbf{v}^{\prime} ; t\right)$ is the Fourier transformation of the probability density in the phase space for the particle transition from the point $\mathbf{v}$ to the point $\mathbf{v}^{\prime}$ for the time $t$. If the particle interactions are neglected, the transition probability density is given by

$$
\begin{aligned}
W_{\mathbf{k}}\left(\mathbf{v}, \mathbf{v}^{\prime} ; t\right) & =e^{-i a\left[\sin \left(\omega_{B} t+\alpha\right)-\sin \alpha\right]-i k_{\|}\|\|^{t}} \\
& \times \delta\left(v_{x}^{\prime}-v_{\perp} \cos \left(\omega_{B} t+\alpha\right)\right) \delta\left(v_{y}^{\prime}+v_{\perp} \sin \left(\omega_{B} t+\alpha\right)\right) \delta\left(v_{\|}^{\prime}-v_{\|}\right),
\end{aligned}
$$

where $a=\frac{k_{\perp} v_{\perp}}{\omega_{B}}$ and the initial velocity is determined as

$$
\mathbf{v}=\left(v_{\perp} \cos \alpha,-v_{\perp} \sin \alpha, v_{\|}\right) .
$$

If the charged particle distribution is axially symmetric with respect to the external magnetic field, the spectral distribution of the Langevin current is as follows:

$$
\left\langle j_{i} j_{j}\right\rangle_{\mathbf{k} \omega}^{0 \alpha}=2 \pi \sum_{\alpha} e_{\alpha}^{2} \int d \mathbf{v} \sum_{n} \Pi_{i j}^{(n \alpha)}\left(v_{\perp}, v_{\|}\right) \delta\left(\omega-k_{\|} v_{\|}-n \omega_{B_{\alpha}}\right) f_{0 \alpha}\left(v_{\perp}, v_{\|}\right)
$$

where the tensor $\Pi_{i j}^{(n)}\left(v_{\perp}, v_{\|}\right)$is defined by

$$
\begin{aligned}
\Pi_{i j}^{(n, \alpha)}\left(v_{\perp}, v_{\|}\right) & =\left\{\begin{array}{l}
\frac{n \omega_{B_{\alpha}}}{k_{\perp}} J_{n}\left(a_{\alpha}\right) \\
-i v_{\perp} J_{n}^{\prime}\left(a_{\alpha}\right) \\
v_{\|} J_{n}\left(a_{\alpha}\right)
\end{array}\right\}\left(\frac{n \omega_{B_{\alpha}}}{k_{\perp}} J_{n}\left(a_{\alpha}\right), i v_{\perp} J_{n}^{\prime}\left(a_{\alpha}\right), v_{\|} J_{n}\left(a_{\alpha}\right)\right), \\
a_{\alpha} & =\frac{k_{\perp} v_{\perp}}{\omega_{B_{\alpha}}} .
\end{aligned}
$$

In the case of a nonequilibrium plasma the dielectric permittivity tensor may be found on the basis of inverting the fluctuation-dissipation relationship [3]:

$$
\varepsilon_{i j}(\omega, \mathbf{k})=\delta_{i j}+4 \pi \sum_{\alpha} \kappa_{i j}^{\alpha}(\omega, \mathbf{k})=\left(1-\sum_{\alpha} \frac{\omega_{p \alpha}^{2}}{\omega^{2}}\right) \delta_{i j}-\frac{2}{\omega^{2}} \sum_{\alpha} \int d \omega^{\prime} \frac{\omega^{\prime} \frac{\partial}{\partial E_{\alpha}}\left\langle j_{i} j_{j}\right\rangle_{\mathbf{k} \omega^{\prime}}^{0 \alpha}}{\omega^{\prime}-\omega-i 0}
$$

where $\frac{\partial}{\partial E_{\alpha}}\left\langle j_{i} j_{j}\right\rangle_{\mathbf{k} \omega^{\prime}}^{0 \alpha}$ is the correlation function averaged over the derivative of the energy distribution:

$$
\begin{aligned}
\omega \frac{\partial}{\partial E_{\alpha}}\left\langle j_{i} j_{j}\right\rangle_{\mathbf{k} \omega^{\prime}}^{0 \alpha} & =\frac{2 \pi e_{\alpha}^{2}}{m_{\alpha}} \sum_{n} \int d \mathbf{v} \Pi_{i j}^{(n, \alpha)}(\mathbf{v}) \delta\left(\omega-n \omega_{B_{\alpha}}-k_{\|} v_{\|}\right) \\
& \times\left(\frac{n \omega_{B_{\alpha}}}{v_{\perp}} \frac{\partial}{\partial v_{\perp}}+k_{\|} \frac{\partial}{\partial v_{\|}}\right) f_{0 \alpha}\left(v_{\perp}, v_{\|}\right)
\end{aligned}
$$




\section{A. G. SITENKO}

\section{POTENTIAL ELECTRIC FIELD FLUCTUATIONS}

For the potential (longitudinal electric) field, the spectral distribution of the Langevin source and the dielectric permittivity $\mathbf{B}_{0}$ are given by

$$
\begin{aligned}
& \left\langle\delta n^{2}\right\rangle_{\mathbf{k} \omega}^{0}=2 \pi \sum_{n} \int d \mathbf{v} J_{n}^{2}\left(\frac{k_{\perp} v_{\perp}}{\omega_{B}}\right) \delta\left(\omega-n \omega_{B}-k_{\|} v_{\|}\right) f_{0}\left(v_{\perp}, v_{\|}\right), \\
& \varepsilon(\omega, \mathbf{k})=1+\sum_{\alpha} \frac{4 \pi e^{2}}{m k^{2}} \sum_{n} \int d \mathbf{v} \frac{J_{n}^{2}\left(\frac{k_{\perp} v_{\perp}}{\omega_{B}}\right)}{\omega-n \omega_{B}-k_{\|} v_{\|}+i 0}\left(\frac{n \omega_{B}}{v_{\perp}} \frac{\partial}{\partial v_{\perp}}+k_{\|} \frac{\partial}{\partial v_{\|}}\right) f_{0}\left(v_{\perp}, v_{\|}\right) .
\end{aligned}
$$

For the strong external magnetic field, in the low-frequency range $\left(\omega \ll \omega_{B_{i}}\right)$ the terms with $n=0$ are significant only in (27) and in the contribution from the second term in the brackets in (28). The contribution from the first term to the latter equation includes all terms with $n \neq 0$. Thus, Eqs. (82) and (83) may be approximated by the formulas [6]:

$$
\begin{gathered}
\left\langle\delta n^{2}\right\rangle_{\mathbf{k} \omega}^{0}=2 \pi \int d \mathbf{v} J_{0}^{2}\left(\frac{k_{\perp} v_{\perp}}{\omega_{B}}\right) \delta\left(\omega-k_{\|} v_{\|}\right) f_{0}\left(v_{\perp}, v_{\|}\right), \\
\varepsilon(\omega, \mathbf{k})=1+4 \pi \sum_{\alpha} \kappa_{\alpha}(\omega, \mathbf{k})=\varepsilon_{0}(\mathbf{k})+\sum_{\alpha} \frac{4 \pi e^{2}}{m k^{2}} \int d \mathbf{v} \frac{J_{0}^{2}\left(\frac{k_{\perp} v_{\perp}}{\omega_{B}}\right)}{\omega-k_{\|} v_{\|}+i 0} k_{\|} \frac{\partial}{\partial v_{\|}} f_{0}\left(v_{\perp}, v_{\|}\right),
\end{gathered}
$$

where

$$
\varepsilon_{0}(\mathbf{k})=1+\sum_{\alpha} \frac{4 \pi e^{2}}{m k^{2}} \int d \mathbf{v}\left\{J_{0}^{2}\left(\frac{k_{\perp} v_{\perp}}{\omega_{B}}\right)-1\right\} \frac{1}{v_{\perp}} \frac{\partial}{\partial v_{\perp}} f_{0}\left(v_{\perp}, v_{\|}\right) .
$$

When particle distributions are Maxwellian with temperatures $T_{\perp}$ and $T_{\|}$with respect to the external magnetic field direction, Eqs. (29) and (30) yield

$$
\begin{aligned}
\left\langle\delta n^{2}\right\rangle_{\mathbf{k} \omega}^{0} & =\sqrt{2 \pi} \frac{n_{0}}{k_{\|} s_{\|}} e^{-\beta} I_{0}(\beta) e^{-z_{\|}^{2}}, \\
\varepsilon(\omega, \mathbf{k}) & =\varepsilon_{0}(\mathbf{k})\left\{1+\frac{1}{\widetilde{a}^{2} k^{2}}\left[1-\varphi\left(z_{\|}\right)+i \sqrt{\pi} z_{\|} e^{-z_{\|}^{2}}\right.\right. \\
& \left.+Z_{i}\left(1-\varphi\left(\mu z_{\|}\right)+i \sqrt{\pi} \mu z_{\|} e^{-\mu^{2} z_{\|}^{2}}\right]\right\},
\end{aligned}
$$

where $Z_{i}$ is the ion charge number,

$$
\begin{aligned}
\varepsilon_{0}(\mathbf{k}) & =1+\sum_{\alpha} \frac{1}{a_{\perp}^{2} k^{2}}\left(1-e^{-\beta} I_{0}(\beta)\right) \\
a_{\perp}^{2} & =\frac{T_{\perp}}{4 \pi n_{0}^{2}}, \quad a_{\|}^{2}=\frac{T_{\|}}{4 \pi n_{0}^{2}}, \quad \widetilde{a}^{2}=\frac{\varepsilon_{0}(\mathbf{k})}{\varepsilon^{-\beta} I_{0}(\beta)} a_{\|}^{2},
\end{aligned}
$$

$$
\beta=\frac{k_{\perp}^{2} T_{\perp}}{m \omega_{B}^{2}}, \quad s_{\|}^{2}=\frac{T_{\|}}{m}, \quad z_{\|}=\frac{1}{\sqrt{2} k_{\|}} \frac{\omega}{s_{\|}} .
$$

We note that the spectral distribution of fluctuation sources in this case (strong magnetic field) is determined by the longitudinal temperature only. The correlation function for the electron density fluctuations in the case of potential interactions between particles takes the form

$$
\begin{aligned}
\left\langle\delta n_{e}^{2}\right\rangle_{\mathbf{k} \omega} & =\left|1-\frac{4 \pi \kappa_{e}(\omega, \mathbf{k})}{\varepsilon(\omega, \mathbf{k})}\right|^{2}\left\langle\delta n_{e}^{2}\right\rangle_{\mathbf{k} \omega}^{0} \\
& +\left|\frac{4 \pi \kappa_{e}(\omega, \mathbf{k})}{\varepsilon(\omega, \mathbf{k})}\right|^{2} Z_{i}^{2}\left\langle\delta n_{i}^{2}\right\rangle_{\mathbf{k} \omega}^{0}
\end{aligned}
$$

Here, plasma discreteness reveals itself in the spontaneous density fluctuations $\left\langle\delta n_{e}^{2}\right\rangle_{\mathbf{k} \omega}^{0}$ and $\left\langle\delta n_{i}^{2}\right\rangle_{\mathbf{k} \omega}^{0}$, due to the random motion of individual non-interacting particles. The factors near the correlation function $\left\langle\delta n_{e}^{2}\right\rangle_{\mathbf{k} \omega}^{0}$ and $\left\langle\delta n_{i}^{2}\right\rangle_{\mathbf{k} \omega}^{0}$ in (31) govern plasma polarization by the shielding cloud of electrons and ions around the test particle. Comparing these equations with those for a non- 
magnetized plasma, we observe that these equations are related by the scaling transformation $z \rightarrow z_{\|}, a^{2} \rightarrow \widetilde{a}^{2}$, $n_{0} \rightarrow \widetilde{n}_{0} \equiv n_{0} e^{-\beta} I_{0}(\beta)$ and $\kappa(\omega, \mathbf{k}) \rightarrow \kappa_{0}(k)+\kappa(\omega, \mathbf{k})$. This makes it possible to reproduce the above analysis using the appropriate results for a nonmagnetized plasma.

\section{COLLECTIVE FLUCTUATIONS IN NONEQUILIBRIUM PLASMAS}

The spectral distributions of electric and magnetic fields fluctuations in the vicinity of the eigenoscillation frequencies in the transmittance range for a nonequilibrium plasma reduce to the form:

$$
\begin{aligned}
\left\langle E_{i} E_{j}\right\rangle_{\mathbf{k} \omega} & =\frac{8 \pi^{2}}{\omega} \widetilde{T}(\omega, \mathbf{k}) e_{i}^{*} e_{j} S p \lambda \delta\left(\Lambda^{\prime}\right), \\
\left\langle B_{i} B_{j}\right\rangle_{\mathbf{k} \omega} & =\frac{8 \pi^{2}}{\omega} \widetilde{T}(\omega, \mathbf{k}) \eta^{2} b_{i}^{*} b_{j} S p \lambda \delta\left(\Lambda^{\prime}\right),
\end{aligned}
$$

where $\widetilde{T}(\omega, \mathbf{k})$ is the effective temperature

$$
\tilde{T}(\omega, \mathbf{k})=\frac{2 \pi^{2}}{\omega} \frac{\lambda_{k l}}{\Lambda^{\prime \prime}}\left\langle j_{k} j_{l}\right\rangle_{\mathbf{k} \omega}^{0}
$$

We note that the effective temperature $\tilde{T}(\omega, \mathbf{k})$ depends on the frequency and the wave vector. It is obvious that the value of the effective temperature for the frequency equal to the eigenoscillation frequency should be regarded as the temperature of the relevant eigenoscillations,

$$
T_{A} \equiv \tilde{T}\left(k v_{A} \cos \vartheta, k\right), \quad T_{S} \equiv \tilde{T}\left(k v_{A}, k\right)
$$

The values of $T_{A}$ and $T_{S}$ can differ considerably. The condition of plasma stability with respect to collective excitations reduces to the requirement that the damping rate $\gamma_{A, S}$ of the relevant collective excitation must be greater than zero. The effective temperature of relevant excitations grows infinite at the stability boundary.

The spectral distribution of incoherent fluctuations of the magnetic field is maximum for zero frequency. The ratios of the intensities of these spectral distributions for zero frequencies to the quantities for an equilibrium plasma, may be regarded as effective temperatures of the relevant incoherent fluctuations, i.e.,

$$
\begin{aligned}
T_{A}^{N} & =8 \sqrt{6 \pi} \sqrt{\frac{M}{m}} \frac{1}{q^{2}} \frac{\omega_{B_{i}}^{2}}{\omega_{P_{i}}} \frac{s}{v_{A}} \frac{\left\langle j_{1}^{2}\right\rangle_{\mathbf{k} \omega}^{(0)}}{k v_{A}} \frac{1}{\sin ^{2} \vartheta \cos \vartheta} \\
T_{S}^{N} & =\sqrt{6 \pi} \sqrt{\frac{M}{m}} \frac{\omega_{B_{i}}^{2}}{\omega_{P_{i}}} \frac{\left\langle j_{2}^{2}\right\rangle_{\mathbf{k} \omega}^{(0)}}{k s} \frac{\cos \vartheta}{\sin ^{2} \vartheta}
\end{aligned}
$$

We note that effective temperatures of incoherent fluctuations are always finite, as distinct from the temperatures of collective Alfven and magnetosonic fluctuations. Thus, it is convenient to describe the state of a nonequilibrium plasma in terms of the sets of temperatures $T_{A}$, $T_{S}$ and $T_{A}^{N}, T_{S}^{N}$; the diagnostics of states of a nonequilibrium plasma with strong external magnetic field may be reduced to the calculation of these temperatures.

\section{FLUCTUATIONS IN TURBULENT PLASMAS}

We consider plasmas with developed turbulence and assume that there occur large-scale turbulent pulsations. This means that microscopic motion of noninteracting particles reduces to the motion of particles under the influence of the field averaged over a small macroscopic volume, and the stochastic motion of the latter volume. We assume thermal motion of individual particles and chaotic turbulent large-scale motions to occur independently. Therefore, the transition probability density for a turbulent system is given by

$$
W_{\mathbf{k} \omega}^{T}\left(\mathbf{v}, \mathbf{v}^{\prime}\right)=\int \frac{d \omega^{\prime}}{2 \pi} \int d \mathbf{v}^{T} P_{\mathbf{k} \omega-\omega^{\prime}}\left(\mathbf{v}^{T}\right) W_{\mathbf{k} \omega^{\prime}}\left(\mathbf{v}, \mathbf{v}^{\prime}+\mathbf{v}^{T}\right)
$$

where $P_{\mathbf{k} \omega}\left(\mathbf{v}^{T}\right)$ is the factor determined by the stochastic Brownian motion of small macroscopic volumes. If the elementary volume is involved in the diffusion-drift motion [6], then

$$
P_{\mathbf{k} \omega} \equiv \int d \mathbf{v}^{T} P_{\mathbf{k} \omega}\left(\mathbf{v}^{T}\right)=\frac{2 k^{2} D}{\left(\omega-\mathbf{k} \mathbf{u}_{D}\right)^{2}+k^{4} D^{2}},
$$

where $\mathbf{u}_{D}$ is the drift velocity and $D$ is the diffusion coefficient. The more pragmatic model [7] one imagines that small macroscopic plasma volumes move chaotically across the magnetic field and the characteristic function is a Gaussian 


$$
P_{\mathbf{k} \omega}=\frac{\sqrt{2 \pi}}{\gamma_{F}} \exp \left\{\frac{\left(\omega-\mathbf{k u}_{D}\right)^{2}}{2 \gamma_{F}^{2}}\right\}
$$

where the mean drift velocity $\mathbf{u}_{D}$ and the root mean square velocity $u$, associated with fluid-like motion, determine the Doppler frequency $\omega_{F}=\mathbf{k} \mathbf{u}_{D}$ (at which the spectrum has its maximum) and the spectral width $\gamma_{F}=k_{\perp} u$, respectively.

The spectral distribution of the Langevin current for a turbulent system is defined by

$$
\left\langle j_{i} j_{j}\right\rangle_{\mathbf{k} \omega}^{T}=\int \frac{d \omega^{\prime}}{2 \pi} P_{\mathbf{k} \omega-\omega^{\prime}}\left\langle j_{i} j_{j}\right\rangle_{\mathbf{k} \omega}^{0}
$$

The dielectric permittivity tensor for a turbulent plasma is determined by the formula (26) in which the correlation function $\left\langle j_{i} j_{j}\right\rangle_{\mathbf{k} \omega}^{0}$ has to be changed to $\left\langle j_{i} j_{j}\right\rangle_{\mathbf{k} \omega}^{T}$. In the case of potential field the spectral distribution of the fluctuation source and the electric susceptibility for a turbulent plasma are given by

$$
\begin{aligned}
\left\langle\delta n^{2}\right\rangle_{\mathbf{k} \omega}^{T} & =\int \frac{d \omega^{\prime}}{2 \pi} P_{\mathbf{k} \omega-\omega^{\prime}}\left\langle\delta n^{2}\right\rangle_{\mathbf{k} \omega^{\prime}}^{0}, \\
\kappa^{T}(\omega, \mathbf{k}) & =\int \frac{d \omega^{\prime}}{2 \pi} \widetilde{P}_{\mathbf{k} \omega-\omega^{\prime}} \frac{\omega^{\prime 2}}{\omega^{2}} \kappa\left(\omega^{\prime}, \mathbf{k}\right) .
\end{aligned}
$$

Making use of the characteristic function (38), we find the spectral distribution for spontaneous fluctuations [7]:

$$
\begin{aligned}
\left\langle\delta n^{2}\right\rangle_{\mathbf{k} \omega}^{T} & =\sqrt{\frac{2 \pi}{C}} \frac{\tilde{n}_{0}}{\gamma_{T}} e^{-\frac{\left(\omega-\omega_{F}\right)^{2}}{2 C \gamma_{T}^{2}}} \\
\gamma_{T} & =k_{\|} s_{\|}, \quad \tilde{n}_{0}=n_{0} e^{-\beta} I_{0}(\beta), \\
\beta & =\frac{k_{\perp}^{2} s_{\perp}^{2}}{\omega_{B_{i}}^{2}}, \quad C=1+\left(\frac{k_{\perp}}{k} \frac{u}{s_{\|}}\right)
\end{aligned}
$$

and the dielectric permittivity for an electron plasma

$$
\varepsilon(\omega, \mathbf{k})=\varepsilon(\mathbf{k})\left\{1+\frac{1}{C} \frac{1}{\widetilde{a}^{2} k^{2}}\left[1-\varphi\left(\frac{\omega-\omega_{F}}{\sqrt{2 C} \gamma_{T}}\right)+i \sqrt{\pi} \frac{\omega-\omega_{F}}{\sqrt{2 C} \gamma_{T}} e^{-\frac{\left(\omega-\omega_{F}\right)^{2}}{2 C \gamma_{T}^{2}}}\right]\right\}
$$

The shape parameter $C$ changes in the range from unity to infinity, as it depends on the ratio between the magnitudes of the wavevector perpendicular and parallel components and the ratio between the root mean square turbulent velocity and the particle thermal velocity. The spectral distribution of electron density fluctuations for a turbulent plasma is related to result for a nonmagnetized plasma by the scaling transformations:

$$
T \rightarrow C T \geq T, \quad \omega \rightarrow \frac{\omega-\omega_{F}}{\sqrt{C}}, \quad a^{2} \rightarrow C \widetilde{a}^{2} .
$$

The characteristic length scale, which separates incoherent and collective fluctuations according to $C \widetilde{a}^{2} k^{2} \varepsilon(\mathbf{k}) \sim$ 1 depends on the effects of fluid motion $(C>1)$, particle polarization drift $(\varepsilon(\mathbf{k})>1)$ and finite Larmor radius. The characteristic scale length is large when these effects are important. The spectra are broader for larger $C$, with collective features being less pronounced. The frequency scale $\sqrt{2 C} \gamma_{T}$ depends on particle thermal motion along the magnetic field and on fluid, and it decreases with $k_{\|}$.

\section{SCATTERED-WAVE-INDUCING CURRENT}

The main problem in calculating the cross-sections of electromagnetic wave scattering in plasmas is to find the current produced by the nonlinear interaction of the incident wave with the fluctuations of electron density and their velocity, and the fluctuations of electric and magnetic fields. This current determines the scattered wave field. The nonlinear constitutive equation for the plasma, and hence the scattered-wave-inducing current may be derived from kinetic or hydrodynamic equations for the electron and ion plasma components. Since the difference between electron and ion masses is very large, the consideration may be restricted to the electron component only. The results of the fluid-approximation study of nonlinear processes in plasmas, obtained within its applicability range, are shown to be in accordance with the results of the kinetic treatment. In the cold plasma, when thermal effects in the dispersion of incident and scattered waves may be completely disregarded, the scatteredwaves-inducing current is given by the expression [3]:

$$
\begin{aligned}
\mathbf{J}_{\mathbf{k} \omega} & =-i\left\{\frac{\omega_{0}}{n_{0}} \delta n_{\mathbf{q} \Delta \omega} \hat{\kappa}\left(\omega_{0}\right) \mathbf{E}^{0}+\delta \mathbf{u}_{\mathbf{q} \Delta \omega}\left(\mathbf{k}_{0} \hat{\kappa}\left(\omega_{0}\right) \mathbf{E}^{0}\right)+\omega \hat{\kappa}(\omega)\left[\frac { 1 } { c } \left(\left[\delta \mathbf{u}_{\mathbf{q} \Delta \omega}, \mathbf{B}_{0}\right]\right.\right.\right. \\
& \left.\left.+\left[\mathbf{u}^{0}, \delta \mathbf{B}_{\mathbf{q} \Delta \omega}\right]\right)-4 \pi \frac{\omega_{0}}{\omega_{P_{e}}^{2}}\left(\left(\mathbf{k}_{0} \delta \mathbf{u}_{\mathbf{q} \Delta \omega}\right) \hat{\kappa}\left(\omega_{0}\right) \mathbf{E}^{0}+\delta \mathbf{u}_{\mathbf{q} \Delta \omega}\left(\mathbf{q} \hat{\kappa}\left(\omega_{0}\right) \mathbf{E}^{0}\right)\right]\right\}
\end{aligned}
$$


Here we introduced the following denotations: $\omega_{0}$ and $\mathbf{k}_{0}$ are a frequency and a wave vector of the incident wave; $\omega$ and $\mathbf{k}$ are a frequency and a wave vector of the scattered wave; $\Delta \omega=\omega-\omega_{0}$ and $\mathbf{q}=\mathbf{k}-\mathbf{k}_{0} ; \mathbf{E}^{0}$ and $\mathbf{B}^{0}$ are the electric and magnetic fields of the incident wave; $\delta n_{\mathbf{q} \Delta \omega}$ and $\delta \mathbf{u}_{\mathbf{q} \Delta \omega}$ are fluctuations of the electron density and velocity; $\delta \mathbf{B}_{\mathbf{q} \Delta \omega}$ is fluctuations of the magnetic field; $\hat{\kappa}(\omega)$ is the dielectric permittivity tensor of the cold magnetoactive plasma,

$$
\begin{gathered}
\varepsilon(\omega) \equiv 1+4 \pi \kappa(\omega)=\left(\begin{array}{lll}
\varepsilon_{1} & -i \varepsilon_{2} & 0 \\
i \varepsilon_{2} & \varepsilon_{1} & 0 \\
0 & 0 & \varepsilon_{3}
\end{array}\right), \\
\varepsilon_{1}(\omega)=1-\sum_{\alpha} \frac{\omega_{P_{\alpha}}^{2}}{\omega^{2}-\omega_{B_{\alpha}}^{2}}, \quad \varepsilon_{2}(\omega)=\sum_{\alpha} \frac{\omega_{B_{\alpha}}}{\omega} \frac{\omega_{P_{\alpha}}^{2}}{\omega^{2}-\omega_{B_{\alpha}}^{2}}, \\
\varepsilon_{3}(\omega)=1-\sum_{\alpha} \frac{\omega_{P_{\alpha}}^{2}}{\omega^{2}} .
\end{gathered}
$$

Using the linear connections between fluctuations of physical quantities in a plasma and the linearity of incident wave, we can present (43) in the form:

$$
J_{i}(\omega, \mathbf{k})=-i \omega \kappa_{i j}(\omega)\left\{\alpha_{j k} \frac{\delta n_{\mathbf{q} \Delta \omega}}{n_{0}}+\gamma_{j k l} \frac{\delta B_{l \mathbf{q} \Delta \omega}}{B_{0}}\right\} E_{k}^{0},
$$

where the fluctuations of all quantities are expressed in terms of the longitudinal fluctuations of charge density and the transversal magnetic fluctuations. Let us consider the scattering of waves with a small change of frequency $\left(\Delta \omega \ll \omega_{0}\right)$. In this case, we can use the approximate expression

$$
\begin{aligned}
\alpha_{j k} & \approx \delta_{j k}-\frac{4 \pi}{q^{2}} q_{j} k_{0 l} \kappa_{l k}\left(\omega_{0}\right), \\
\gamma_{j k l} & \approx-4 \pi \frac{\omega_{0} \omega_{B}}{\omega_{P}^{2}} \epsilon_{j m l} \kappa_{m k}\left(\omega_{0}\right), \quad \Delta \omega \ll \omega_{0} .
\end{aligned}
$$

Ordinary and extraordinary electromagnetic waves with equal frequencies but different refraction index and polarization can propagate in a plasma with the external magnetic field. The polarization vectors of the ordinary and extraordinary waves $\mathbf{e}_{0}$ and $\mathbf{e}_{x}$, respectively, satisfy the orthogonality condition

$$
\varepsilon_{i j} e_{0 i} e_{x j}^{*}=0
$$

For the waves that propagate along the magnetic field $(\vartheta=0)$,

$$
\eta_{0, x}^{2}=\varepsilon_{1} \mp \varepsilon_{2}, \quad \mathbf{e}_{0, x}=(1, \mp i, 0),
$$

in the other words, these waves are transversal with the circular polarization. For the waves that propagate in the direction perpendicular to the magnetic field $\left(\vartheta=\frac{\pi}{2}\right)$ :

$$
\begin{aligned}
\eta_{0}^{2}=\varepsilon_{3}, \quad \mathbf{e}_{0} & =(0,0,1) ; \quad \eta_{x}^{2}=\varepsilon_{1}-\frac{\varepsilon_{2}^{2}}{\varepsilon_{1}}, \\
\mathbf{e}_{x} & =\left(1,-i \frac{\varepsilon_{1}}{\varepsilon_{2}}, 0\right) .
\end{aligned}
$$

If the incident electromagnetic wave propagates along the magnetic field $\mathbf{k}_{0} \| \mathbf{B}_{0}$, then $\alpha_{i j} \rightarrow \delta_{i j}$; at the same time the scattering of waves on the longitudinal charge density fluctuations takes place. Because of the orthogonality of the polarization vectors $\mathbf{e}_{0}$ and $\mathbf{e}_{x}$, the wave conversion on the charge density fluctuations is impossible.

If the incident wave (let $\mathbf{e}_{0}$ ) propagates perpendicularly to the direction of magnetic field $\mathbf{k}_{0} \perp \mathbf{B}_{0}$, then $\mathbf{k}_{0} \hat{\kappa}\left(\omega_{0}\right) \mathbf{e}_{0}=0$ and again we get $\alpha_{j k}=\delta_{j k}$. Therefore, the wave conversion or transformation of the ordinary electromagnetic wave in the extraordinary one occurs due to the interaction with the transversal magnetic fluctuations only.

\section{ELECTROMAGNETIC WAVE SCATTERING CROSS-SECTION}

The general expression for the differential crosssection of electromagnetic wave scattering in magnetoactive plasmas was derived in [3] from the hydrodynamic equations. In the case of electromagnetic wave scattering by electron density fluctuations, the differential crosssection is given by

$$
d \Sigma=\frac{1}{2 \pi}\left(\frac{e^{2}}{m c^{2}}\right)^{2} \frac{\omega_{0}^{2} \omega^{2}}{\omega_{P_{e}}^{4}} R\left|\xi^{2}\right|\left\langle\delta n_{e}^{2}\right\rangle_{\mathbf{q} \Delta \omega} d \omega d 0
$$

where

$R=\frac{\eta^{3}}{\eta_{0}\left(\left|\mathbf{e}_{0}\right|^{2}-\frac{\left|\mathbf{e}_{0} \mathbf{k}_{0}\right|^{2}}{k_{0}^{2}}\right) e_{i}^{*} e_{i j} e_{j}}, \quad \xi=e_{i}^{*}\left(\varepsilon_{i j}^{0}-\delta_{i j}\right) e_{j}^{0}$

This formula determines the plasma parameters (density, temperature, etc.) in terms of observed electromagnetic wave scattering spectra.

\section{ELECTROMAGNETIC WAVE CONVERSION CROSS-SECTION}

The differential cross-section of electromagnetic wave conversion under scattering can be calculated in the same manner as in [3]. We restrict the consideration to the electromagnetic wave conversion with a small frequency shift $\left(\Delta \omega \ll \omega_{0}\right)$, then the differential cross-section of conversion is described by the expression 


\section{A. G. SITENKO}

$$
d \Sigma=\frac{1}{2 \pi} \frac{\omega^{4}}{c^{4} B_{0}^{2}} R \sum_{l, l^{\prime}}\left(e_{i}^{*} \kappa_{i j}(\omega) \gamma_{j k l} e_{k}^{0}\right)\left(e_{i}^{*} \kappa_{i j}(\omega) \gamma_{j k l^{\prime}} e_{k}^{0}\right)^{*}\left\langle\delta B_{l} \delta B_{l^{\prime}}^{*}\right\rangle_{\mathbf{q} \Delta \omega} d \omega d 0
$$

We note that

$$
\left\langle\delta B_{l} \delta B_{l^{\prime}}^{*}\right\rangle_{\mathbf{q} \Delta \omega}=\delta_{l l^{\prime}}\left\langle\delta B_{l}^{2}\right\rangle_{\mathbf{q} \Delta \omega},
$$

and make use of the expression for the coefficient $\gamma_{j k l}$, then the differential cross-section reduces to the form

$$
d \Sigma=32 \pi^{2}\left(\frac{e^{2}}{m c^{2}}\right)^{2} R \frac{n_{0}}{m c^{2}} \frac{\omega^{6}}{\omega_{P_{e}}^{6}} \sum_{l}\left|e_{i}^{*} \kappa_{i j}(\omega) \epsilon_{j n l} \kappa_{m k}\left(\omega_{0}\right) e_{k}^{0}\right|^{2}\left\langle\delta B_{l}^{2}\right\rangle_{\mathbf{q} \Delta \omega} d \omega d 0 .
$$

We consider the case when the incident ordinary electromagnetic wave is converted into an extraordinary electromagnetic wave. Suppose the process occurs in the plane perpendicular to the external magnetic field $\mathbf{B}_{0}$. The polarization vector of the ordinary wave $\mathbf{e}_{0} \equiv \mathbf{e}^{0}=$ $(0,0,1)$ is directed along the magnetic field $\mathbf{B}_{0}$, hence the azimuthal angle of the wave vector $\mathbf{k}_{0}$ of the incident wave is insignificant. The polarization vector of the extraordinary wave, $\mathbf{e}_{x}$, lies in the plane perpendicular to $\mathbf{B}_{0}$ and depends on the azimuthal angle $\varphi$ of the wave vector $\mathbf{k}$, i.e.,

$$
\mathbf{e}_{x} \equiv \mathbf{e}=\left(\cos \varphi+i \frac{\varepsilon_{1}}{\varepsilon_{2}} \sin \varphi, \sin \varphi-i \frac{\varepsilon_{1}}{\varepsilon_{2}} \cos \varphi, 0\right)
$$

We consider the case when the wave vector change under conversion $\mathbf{q}=\mathbf{k}-\mathbf{k}_{0}$ is small, then we can assume that $\mathbf{q} \perp \mathbf{k}$. We choose the coordinate system in a way that the vector $\mathbf{q}$ is directed along the $x$-axis, then the azimuthal angle of the converted wave vector is $\varphi=-\frac{\pi}{2}$. Therefore, the polarization vector of the converted wave is given by

$$
\mathbf{e}_{x} \equiv \mathbf{e}=\left(-i \frac{\varepsilon_{1}}{\varepsilon_{2}},-1,0\right)
$$

(We do not normalize the converted wave polarization vector since the cross-section (50) does not depend on the normalization condition). Thus we obtain an expression for the differential cross-section of the electromagnetic wave conversion in magnetized plasma, i.e.,

$$
\begin{aligned}
d \Sigma_{0 \rightarrow x} & =\frac{1}{8 \pi^{2}}\left(\frac{e^{2}}{m c^{2}}\right)^{2} R \frac{n_{0}}{m c^{2}}\left\{\frac{\omega^{2}}{\omega_{P_{e}}^{2}}\left\langle\delta B_{1}^{2}\right\rangle_{\mathbf{q} \Delta \omega}\right. \\
& \left.+\frac{\left(\omega^{2}+\omega_{P_{e}}^{2}\right)^{2}}{\omega_{P_{e}}^{2} \omega_{B_{e}}^{2}}\left\langle\delta B_{2}^{2}\right\rangle_{\mathbf{q} \Delta \omega}\right\} d \omega d 0
\end{aligned}
$$

where

$$
R=\frac{\eta^{3}}{\eta_{0} e_{i}^{*} \varepsilon_{i j} e_{j}}=\sqrt{\frac{\varepsilon_{1}^{2}-\varepsilon_{2}^{2}}{\varepsilon_{1} \varepsilon_{2}}} \frac{\varepsilon_{2}^{2}}{\varepsilon_{1}^{2}}
$$

The first term within the curly braces in (51) describes the conversion of an ordinary electromagnetic wave into an extraordinary one due to the interaction with magnetosonic fluctuation excitations in the plasma, the second term is associated with Alfven fluctuation excitations. We note that, for an equilibrium plasma, the integral contribution of Alfven excitations in the conversion is greater than the contribution of magnetosonic excitations,

$$
\frac{\Sigma^{A}}{\Sigma^{S}}=\frac{\left(\omega^{2}+\omega_{P_{e}}^{2}\right)^{2}}{\omega^{2} \omega_{B_{e}}^{2}}>1
$$

In nonequilibrium plasmas, this ratio can be both greater and smaller than one for various plasma states. In the case of conversion by incoherent magnetic fluctuations with very small frequency shift, the value of this ratio is determined by the inverse ratio of the Langevin sources, i.e.,

$$
\frac{\Sigma^{A}}{\Sigma^{S}}=\frac{\left\langle j_{1}^{2}\right\rangle_{\mathbf{k} 0}^{0}}{\left\langle j_{2}^{2}\right\rangle_{\mathbf{k} 0}^{0}}
$$

It should be noted that the analysis of experimental data must take into account the fluid-like turbulent motion in the plasma which can considerably influence the character of the converted wave spectrum, similarly to the effect of such motions on the spectra of wave scattering by particle density fluctuations.

\section{CONCLUSIONS}

The main purpose of this review is to consider the low-frequency properties of a plasma with strong magnetic field, in particular, the specifics of collective excitations, dispersion, polarization, and damping of Alfven 
and magnetosonic waves. Low-frequency fluctuations of charge density, longitudinal and transverse electric field, and magnetic field in a magnetized equilibrium plasma are considered in detail; spectral distributions of such fluctuations are found; incoherent fluctuations caused by chaotic motion of individual particles are separated as well as the collective fluctuations associated with Alfven and magnetosonic plasma excitations. In the range of very small frequencies (much smaller than the ion cyclotron frequency) the Alfven and magnetosonic longwave fluctuations are manifested in different components of the fluctuation spectral distribution tensor, i.e., Alfven and magnetosonic fluctuations are separated due to different characters of polarization of these excitations. The positions of the maxima of fluctuation spectra are determined by the frequencies of relevant plasma eigenoscillations. As the wave number increases, the maxima of fluctuation spectra are shifted to the ion cyclotron frequency range and separation of Alfven and magnetosonic waves disappears; the maxima associated with Alfven and magnetosonic fluctuations are manifested in all components of the spectral distribution; the magnetosonic maximum rapidly approaches the ion cyclotron frequency as the wave number grows. In the vicinity of the ion cyclotron frequency, a cyclotron resonance occurs which leads to the damping of collective excitations. In the ion cyclotron frequency range, incoherent cyclotron fluctuations occur; their spectral distribution is also found in the paper. Much attention is paid to the fluctuations in nonequilibrium plasmas. The Langevin fluctuation sources are introduced in order to find the effective temperatures which are responsible for the collective fluctuation levels for the electric and magnetic fields. The effective temperatures are found as well for the incoherent magnetic field fluctuations associated with Alfven and magnetosonic excitations. The temperatures thus introduced make it possible to describe the nonequilibrium plasma states quantitatively. Fluctuations are also considered in a plasma with large-scale turbulent pulsations. The influence of such fluid-like chaotic motions on the fluctuation spectra is analyzed. Such motions must be taken into account when the plasma state is studied in terms of fluctuation spectra and electromagnetic wave scattering in the plasma. Electromagnetic wave scattering is one of the most efficient methods of plasma diagnostics both in controlled fusion devices and in ionospheric and space plasmas. The study of electromagnetic wave conversion resulting from wave-plasma interaction provides additional possibilities to obtain information on the plasma state, in particular the character of magnetic field fluctuations.
[1] A. I. Akhiezer, I. G. Prohoda, A. G. Sitenko, Sov. Phys. JETP 6, 576 (1958).

[2] A. G. Sitenko, K. N. Stepanov, Sov. Phys. JETP 4, 512 (1957).

[3] A. G. Sitenko, Electromagnetic Fluctuations in Plasma (Academic Press, New York, 1967).

[4] K. N. Stepanov, Sov. Phys. JETP 7, 892 (1958); 11, 192
(1960).

[5] A. G. Sitenko, A. V. Kharchenko, Plasma Phys. Control. Fusion 41, 399 (1999).

[6] A. G. Sitenko, A. G. Zagorodny, Ukr. Fiz. Zh. 40, 390 (1995).

[7] P. P. Sosenko, N. Maafa, D. Gresillon, J. Atmos. Terr. Phys. 58, 1047 (1996).

\title{
РОЗСІЯННЯ ТА КОНВЕРСІЯ ЕЛЕКТРОМАГНЕТНИХ ХВИЛЬ НА НИЗЬКОЧАСТОТНИХ КОЛЕКТИВНИХ ФЛЮКТУАЩЯХ У НАМАГНЕТОВАНІӤ ПЛАЗМІ
}

\author{
О. Г. Ситенко \\ Інститут теоретичной фізики ім. М. М. Боголюбова, \\ вул. Метрологічна, 14-Б, Киӥв, 03143, Украӥна \\ E-mail: ositenko@gluk.apc.org
}

\begin{abstract}
Розглянуто розсіяння електромагнетних хвиль у плазмі за наявности сильного зовнішнього магнетного поля й обговорено можливість його використання для діягностики плазми. Розсіяння пов'язане як з повздовжніми флюктуаціями поля (флюктуаціями густини заряду), так і з поперечними електромагнетними флюктуаціями. Розсіяння електромагнетних хвиль на магнетних флюктуаціях супроводжується конверсією хвиль. Розраховані спектри флюктуацій магнетного та електричного полів у низькочастотній області для плазми за наявности сильного зовнішнього магнетного поля. Виділені некогерентні флюктуапії та флюктуапї зумовлені збудженням колективних мод (Альфвенових та магнетозвукових хвиль). Досліджено спектральні розподіли флюктуаџій електричного та магнетного полів у нерівноважній плазмі. Обговорено також флюктуапї в плазмі за наявности великомасштабних турбулентних пульсапій. Розраховано переріз розсіяння хвиль і визначено умови, за яких конверсія хвиль $є$ домінуючим процесом. Вивчено особливості спектрального розподілу розсіяних хвиль залежно від параметрів, що характеризують стан плазми.
\end{abstract}

